\title{
En busca del proyecto ideal. Una orientación para la exploración de proyectos y su formulación
}

\section{Víctor Julio Sinuco Moreno*}

In search of the ideal project. An orientation for projects' exploration and formulation

À la recherche du projet idéal. Une arientation pour l'exploration de projets et leur formulation

A procura do projeto ideal. Uma arientação para a explaraçãa de projetos e sua formulaçãa

"Especialización en Gestión para el Desarrollo Empresarial, Universidad Santo Tomás; Especialización en Gerencia de Proyectos de Informática, Universidad del Rosario; Ingeniero de Sistemas con énfasis en Software profesional, Universidad Antonio Nariño. Director de Sistemas y Tecnología en Colpensiones; Analista/Gerente de proyectos en Cardif. 


\section{RESUMEN}

Este artículo le brindará la posibilidad de visualizar algunas notas del autor, la proposición de un par de metodologías para la gestión de proyectos, la sugerencia de algunos proyectos y la mención de ciertas guías que le permitirán ubicar de forma estratégica, geográfica, histórica $y$ financiera un proyecto. Dentro de los elementos a ser considerados se encuentra: usar como guía de ubicación el Plan de Desarrollo Territorial de su municipio, departamento o país. Reconocer o usar el análisis de las cadenas productivas de diferentes productos, que ofrece el Departamento Nacional de Planeación. Usar el documento del Consejo Nacional de Política Económica y Social (CONPES) 3527 - Departamento Nacional de Planeación. Visualizar las oportunidades que tienen los Tratados de Libre Comercio (TLC) para Colombia. Los aspectos que deberá vigilar, son: alinearse con la legislación para garantizar una sana relación entre bien público, ejercicio profesional e interés particular; la ética de la profesión del líder, el Sponsor o cualquier otro interesado influyente en el proyecto; definir un plan; delimitar de forma adecuada el proyecto, para tal fin, usar del SENA un documento focalizado en definir taxonomía de verbos. Las metodologías propuestas para buscar, formular y ejecutar proyectos, son Project Management Institute (PMI $\left.{ }^{\circledast}\right)$ y Marco Lógico. Se recomienda tener precaución con el análisis financiero, las regulaciones y la gestión de riesgos. Dentro de los temas para proyectos propuestos se encuentran: la Responsabilidad Social Empresaria (RSE); el "Pensamiento Estratégico" para hacer un análisis estructural del sector estratégico; la teoría de restricciones (TOC - "Theory of Constraints"); la gestión de conocimiento; gestión de la información; ver la necesidad del desarrollo personal; el liderazgo; el Balanced Scorecard; la seguridad informática, Business Continuity Plan $(B C P)$, contingencia y sistemas de gestión integrados.

\section{ABSTRACT}

This article is intended to provide some personal notes of its author, the proposal a couple of methodologies for project management, some projects' suggestions and several guidelines which allow us to develop a project in a strategic, geographical, historical and financial way. There are some elements to be considered: to use the Territorial Development Plan of your region, department or country as a guideline to develop a project; to identify and use the analysis of production chains for different products, that the National Planning Department offers; the use of the 3527 official document of the National Council of Economic and Social Policy (CONPES), National Planning Department; and to identify the opportunities that the Free Trade Agreements (FTA) have for Colombia. The aspects that should be taken into account are : to be aligned with the legislation to guarantee a sane relationship among public affairs, professional exercise and particular interests; the ethics of a leader, sponsor or anyone directly involved in a project, to set up a plan, to broaden and delimit the project goals and the plan itself; for this purpose it is important to use an official document of SENA focused on defining the taxonomy of verbs. The suggested methodologies to explore, formulate and execute projects are PMI (Project Management Institute) and Logical Framework. It is indispensable to be careful when making the financial analysis, the regulations and risk management. The themes for developing projects are: Social Responsibility, the Strategic Thought to make a well-structured analysis of the strategic sector, the Restrictions Theory, Knowledge Management, Information Management, the need for personal development, Leadership, the Balanced Scorecard, Informatics Safety, Business Continuity Plan, Contingency and Integrated Management Systems.

\section{Palabras claves}

\section{Pensamiento estratégico} Mangement Gestión de proyectos

Formulación de proyectos Cadenas productivas

\section{Key words}

Strategic thought Management

Project management Project formulation Production chains 


\section{RESUMÉÉ}

Cet article a pour objectif de mettre en lumière différentes analyses personnelles de l'auteur, de proposer différentes méthodologies de gestion de projets, d'en suggérer certaines et de recommander des guides devant permettre au lecteur de mettre en place son projet sur des bases stratégiques, géographiques, historiques et financières solides. Les éléments à prendre en compte sont: L'utilisation du Guide d'Aménagement du Territoire de votre ville, département ou pays. La prise en compte de l'analyse des chaines productives des différents produits que présente le Département National au Plan. L'utilisation de documents du Conseil National de Politique Economique et Sociale (CONPES) 3527. Département National au Plan. Etre attentif aux opportunités offertes par les Traités de Libre Échange en vigueur avec la Colombie. Les aspects à prendre en considération sont : être en règle avec la législation en vigueur pour garantir une relation adéquate entre le bien publique, l'exercice professionnel, et l'intérêt particulier ; l'éthique professionnelle du gérant, le sponsor ou toute autre partie prenante influente au projet ; définir un plan d'exécution; délimiter correctement le projet et, à cette fin, utiliser un document du SENA définissant la taxonomie des verbes. Les méthodologies proposées pour rechercher, formuler et exécuter des projets sont exposés dans le Cadre Logique et le PMI ${ }^{\circledR}$ (Project Management Institute). L'analyse financière, les circulaires administratives et la gestion des risques doivent être étudiées avec précaution. Les thèmes présentés pour les projets proposés sont : la Responsabilité Sociale Entrepreneuriale (RSE), la "Pensée Stratégique " pour réaliser une analyse structurelle du secteur, la Théorie des Restrictions » (TOC - Theory of Constraints), la gestion de la connaissance, gestion de l'information, développement personnel, leadership, sécurité informatique, Business Continuity Plan (BCP), Contingence, Systèmes de gestion intégrés.

\section{RESUMO}

Este artigo dará a possibilidade de visualizar algumas notas pessoais do autor, a proposta de um par de metodologias para a gestão de projetos, a sugestão de alguns projetos e a menção de certas dicas que lhe permitirão localizar um projeto de maneira estratégica, geográfica, histórica e financeira. Dentro dos elementos a ser considerados estão: usar como guia de localização o Plano de Desenvolvimento Territorial de seu município, departamento ou país. Reconhecer ou usar a análise das cadeias produtivas de diferentes produtos que oferece o Departamento Nacional de Planejamento. Usar o documento do Conselho Nacional de Política Económica e Social (CONPES) 3527 - Departamento Nacional de Planejamento. E 4) visualizar as oportunidades que trazem os Tratados de Livre Comercio (TLC) para a Colômbia. Os aspectos que devem ser vigiados são: conformar-se com a legislação para garantir uma sã relação entre o bem público, o exercício profissional e o interesse particular; a ética da profissão do líder, o patrocinador ou qualquer outro interessado com influência no projeto; definir um plano; delimitar de maneira adequada o projeto e, para este fim, usar um documento do SENA (serviço Nacional de Aprendizagem) focalizado em definir taxonomia de verbos. As metodologias propostas para procurar, formular e executar projetos, são PMI® (Project Management Institute) e Marco Lógico. Recomenda-se tomar cuidado com a análise financeira, as regulações e a gestão de riscos. Dentro dos temas para projetos propostos estão: a Responsabilidade Social Empresarial (RSE); o "Pensamento Estratégico" para fazer uma análise estrutural do setor estratégico; a teoria de restrições (TOC - "Theory of Constraints"); a gestão do conhecimento; gestão da informação; perceber a necessidade de desenvolvimento pessoal; a liderança; o balanced scorecard; a segurança informática, Business Continuity Plan $(B C P)$, contingência; e sistemas de gestão integrados.

\section{Mots clefs \\ Pensé stratégique Management \\ Gestion de projets \\ Formulation de projets \\ Chaines productives.}

\section{Palavras-chave}

Pensamento estratégico Management

Gestão de projetos

Formulação de projetos Cadeias produtivas. 


\section{INTRODUCción}

E $n$ la definición del plan estratégico de una organización, en el cierre de un estudio de pregrado o posgrado, o en otros múltiples tópicos de la vida nos vemos avocados a proponer proyectos o aún más difícil, a formularlos. El presente artículo, como guía orientadora, hará mención de un par de metodologías para la gestión de proyectos, la sugerencia de algunos proyectos y la enumeración de unos consejos que le permitirán ubicar de forma estratégica, geográfica, histórica y financiera un proyecto. Dentro de los elementos a ser considerados se encuentran: usar como guía de ubicación la documentación que los entes gubernamentales generan como producto de la legislación o el apoyo a los diferentes sectores económicos, dentro de los que se encuentran leyes, estudios y mecanismos de estímulo. Sin lugar a dudas, uno de los factores críticos de éxito es una adecuada formulación y dimensión del proyecto; para tal fin se propondrá el uso de herramientas ya existentes que facilitarán la labor. Algunos de los temas para observar son: la responsabilidad social empresaria (RSE); la teoría de restricciones, Theory of Constraints - (TOC) y el liderazgo.

Para cerrar el tema se presenta el planteamiento del autor de este artículo, que ilustra un enfoque integral. En "Buscando el proyecto ideal" usted podrá agotar muchos recursos como consultar el Plan de Desarrollo Bogotá Humana, la Guía de Cadenas Productivas, los documentos del Consejo Nacional de Política Económica y Social (CONPES), los tratados de libre comercio (TLC) y en general, todas las regulaciones del sector económico. Puede además considerar el alinearse con la ética, tener precaución de hacer una buena formulación del proyecto, hacer una adecuada gestión de riesgos, hacer uso de una buena metodología de gestión de proyecto, y estar enfocado en uno muy bueno; sin ambargo, si el insumo esencial no está dado, los resultados no pueden ser garantizados. Por lo anterior se recomienda, antes de continuar saturando a las empresas y a sus colaboradores con proyectos, hacer el proyecto que imprimirá sinergia en los demás proyectos: el de desarrollar el capital humano de la organización, ya que dicho capital genera la ventaja competitiva y de seguro, puede llegar a hacer que dicha ventaja sea sostenible. No se debe olvidar que, se requiere de motivación, generosidad, enfoque a procesos, capital humano, desarrollo personal, enfoque a principios, desarrollo de las dimensiones sociales, físicas, psicológicas, éticas, intelectuales, políticas, etc.

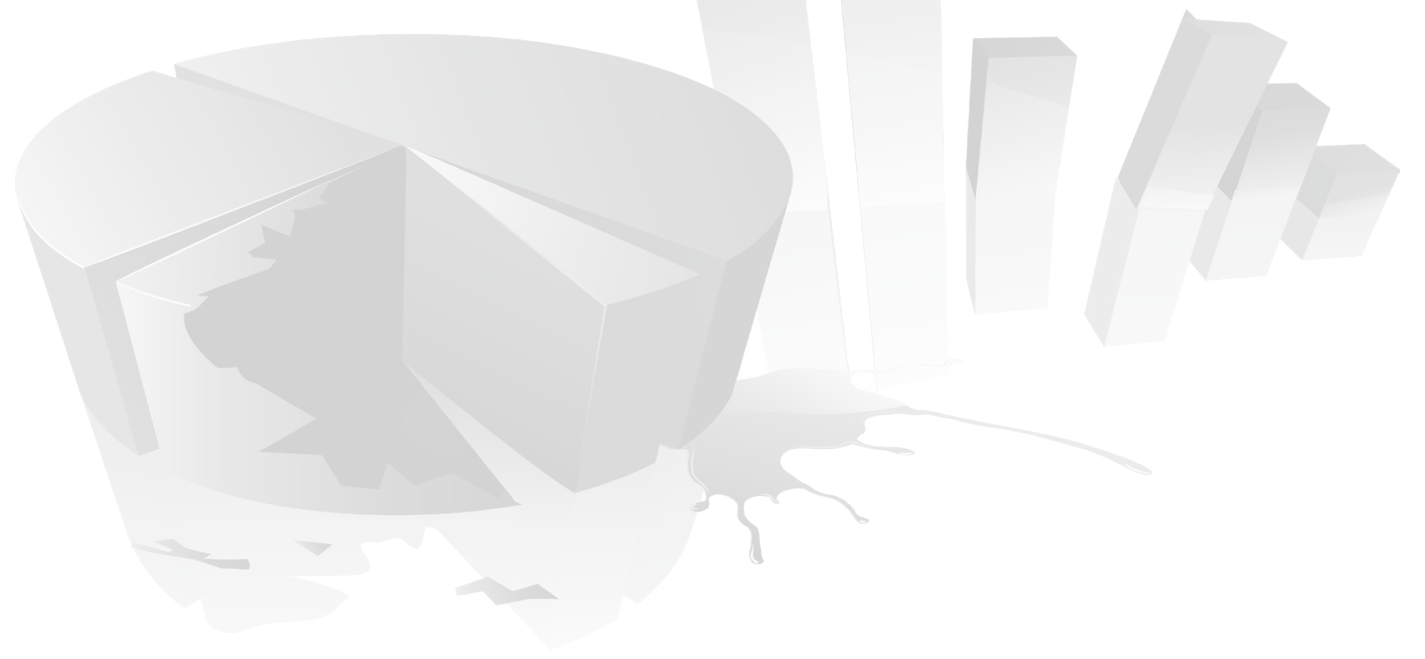




\section{EN BUSCA DEL PROYECTO IDEAL}

Ci usted está leyendo este artículo, de seguro se encuentra frente al fascinante reto de formular un proyecto; probablemente porque tiene un producto o servicio para ofrecer a la sociedad o porque necesita estructurar una tarea

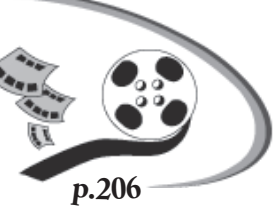
encomendada por su empresa o mejor aún, porque requiere visualizar una población, descubrir la causa de algún problema y plantear una solución. Cualquiera que sea el caso, este documento le brindará unas ideas que le permitirán tener control sobre el bosque y claridad sobre los árboles; su visión será integral.

\subsection{Guía de aspectos a considerar}

Los elementos mencionados a continuación, pueden brindar algunos criterios para definir el marco lógico de un producto, servicio, proyecto o problema; pero también pueden ser herramientas de exploración de oportunidades. Con miras a alinear proyectos con el plan estratégico gubernamental, aprovechar oportunidades y prevenir sorpresas, es de vital importancia tener claro el panorama nacional y regional. Por tal motivo se invita a ubicar los documentos que enmarcan el plan de gobierno de su estado o región, tales como:

\section{- Guía Plan de Desarrollo Bogotá Humana}

Un documento que le brindará un panorama claro de las oportunidades que su región y estado ofrecen, es la guía del plan de desarrollo territorial que para el caso de la capital es la "Guía Plan de Desarrollo Bogotá Humana". La Comisión del Plan del Concejo de la ciudad aprobó el Plan de Desarrollo Bogotá Humana 2012-2014, una hoja de ruta, por el cual se adopta el plan de desarrollo económico, social, ambiental y de obras públicas para Bogotá D. C.

\section{- Guía cadenas productivas}

Herramienta que ofrece las estadísticas de los perfiles sectoriales que son útiles para el análisis de las cadenas productivas. Para profundizar ver el libro Cadenas productivas. Estructura, comercio internacional y protección, publicado por el Departamento Nacional de Planeación en el 2004.

\section{- Guía del Consejo Nacional de Política Económica y Social (CONPES) 3527-2008}

Documento mediante el cual se adopta el plan de desarrollo económico, social, ambiental y de obras públicas para Bogotá D. C. 2012-2016. Bogotá Humana. Emanado por la alta Consejería Presidencial para la Competitividad y la Productividad, del Ministerio de Comercio, Industria y Turismo, el Concejo Privado de Competitividad del Departamento Nacional de Planeación: Gerencia de Competitividad Grupo de Apoyo a la Competitividad y Dirección de Desarrollo Empresarial. El documento aborda temas como la infancia y adolescencia, la salud, la educación, el desarrollo económico y la tecnología.

\section{- Guía de Tratado de Libre Comercio (TLC)}

Los Tratados de Libre Comercio (TLC) son una ventana a las posibilidades o a las amenazas. Es un capítulo obligatorio al momento de hacer estrategia. Para enero del año 2013 los tratados vigentes, según el Ministerio de Comercio, Industria y Turismo, son los que se encuentran publicados en su página $W e b$.

\subsubsection{Regulaciones}

Es aconsejable revisar toda la regulación que exista para su sector económico, como la legislación Web que tiene leyes de propiedad intelectual, ley de accesibilidad, ley antispam, ley de protección de datos; o la legislación educativa, la de 
salud, la del sector financiero, etc. Cualquiera sea su sector económico, este está regulado y es factor clave de éxito el conocer la legislación y cumplirla. Para ello se recomienda revisar los siguientes entes reguladores:

\section{Superintendencia de Industria y Comercio}

Información general sobre esta Institución. Normatividad, publicaciones, notificaciones.

\section{Superintendencia de Servicios Públicos}

Institución encargada de ejercer control sobre las entidades prestadoras de servicios públicos domiciliarios.

\section{Superintendencia de Sociedades}

Información de sociedades, publicaciones, quejas y reclamos. Protección al consumidor. Protección de la competencia. Propiedad industrial.

Y en general la constitución nacional, las leyes, los decretos, las resoluciones, la jurisprudencia, las circulares, el Sistema de Administración de Riesgo Operativo (SARO) y el Sistema de Administración del Riego de Lavado de Activos y de la Financiación del Terrorismo (SARLAFT), y todas las demás normas internacionales.

\subsection{Notas de integración}

Es recomendable que los proyectos estén articulados con el plan institucional o portafolio de proyectos, así como con la institución misma. Para tal fin hay que velar por:

Nota. Alinearse con la legislación para garantizar una sana relación entre bien público, ejercicio profesional e interés particular, garantizando una vida sana al proyecto y a la empresa.

Nota. La ética de la profesión del líder del proyecto, del Sponsor o de cualquier otro interesado influyente deberá ser celosamente guardada, para tener cohesión y coherencia con los compromisos del profesional.

Nota. Al hacer la propuesta y el planteamiento del proyecto, tener precaución con la forma de hacerlo. El mal manejo de los verbos puede crear falsas expectativas y condenar un buen proyecto a la muerte prematura por falta de dimensión apropiada y escalamiento. Para mayor información véase el documento emitido por el SENA: "Taxonomía de verbos".

\section{METODOLOGÍA RECOMENDADA}

$\mathbf{P}$ ara la formulación de un proyecto y para garantizar una sana ejecución, es recomendable no improvisar. Dentro de las múltiples alternativas, dos de las complementarias son:

- Metodología Project Management Institute (PMI $\left.{ }^{\circledR}\right)$ : herramienta fuerte en la ejecución del proyecto que contempla: grupo de procesos de dirección de proyectos, implicando la iniciación, planificación, ejecución, seguimiento, control y cierre. Procesos de un área de conocimiento que cubre integración, alcance, tiempo, costes, calidad, recursos, comunicaciones, riesgos, adquisiciones y gestión de interesados.

Dado el Project Management Body of Knowledge $\left(\mathrm{PMBOK}^{\circledR}\right)$, libro de estándares para la gestión de proyectos) que es desarrollado por el Project Management Institute (PMI), podemos definir los elementos componentes del proyecto, los cuales pueden ser vistos también, como una lista de chequeo que nos determina los documentos a gestionar (tabla 1). 
En busca del proyecto ideal. Una orientación para la exploración de proyectos y su formulación

Bogotá, Pp.138-149

Tabla 1. Lista de chequeo PMBOK ${ }^{\circledR}$

\begin{tabular}{|c|}
\hline Procesos $\mathrm{PMBOK}^{\circledR} 5$ \\
\hline Grupo de procesos de iniciación \\
\hline Grupo de procesos de planificación \\
\hline Grupo de procesos de ejecución \\
\hline Grupo de procesos de seguimiento y control \\
\hline Grupo de procesos de cierre \\
\hline Áreas de conocimiento $\mathrm{PMBOK}{ }^{\circledR} 5$ \\
\hline 1. Gestión de la integración del proyecto \\
\hline $\begin{array}{l}\text { Dados los procesos, esta área de conocimiento permite realzar la identificación, definición, combinación, unificación y coordinació } \\
\text { de los procesos y actividades, para garantizar una integración en condiciones controladas y bajo certidumbre. Las actividades son } \\
\text { desarrollar el acta de constitución del proyecto, desarrollar el plan para la dirección del proyecto, dirigir y gestionar la ejecución de } \\
\text { proyecto, monitorizar y controlar el trabajo del proyecto, realizar el control integrado de cambios y cerrar el proyecto o fase. }\end{array}$ \\
\hline
\end{tabular}

\section{Gestión del alcance del proyecto}

Dada la línea base del alcance, esta permite definir acciones para garantizar su cumplimiento en condiciones efectivas. Implica las acciones: recopilar requisitos, definir el alcance, verificar el alcance, controlar el alcance.

\section{Gestión del tiempo del proyecto}

Determina una serie de actividades y herramientas que permiten cumplir en condiciones predefinidas la línea de tiempo. Las actividades son: definir las actividades, secuenciar las actividades, estimar los recursos de las actividades, estimar la duración de las actividades, desarrollar el cronograma y controlar el cronograma.

\section{Gestión de los costos del proyecto}

Dada la línea base de costes, esta permite estimar, presupuestar y controlar los costes de manera que el seguimiento y control garanticen certidumbre de costos. Las actividades son: estimar los costos, determinar el presupuesto y controlar los costos.

\section{Gestión de la calidad del proyecto}

Determina las acciones y herramientas requeridas para garantizar que, en su integridad, el proyecto se cumpla en condiciones de certidumbre gracias al seguimiento y control. Implica la alineación con los sistemas de gestión de la organización que ejecuta. Las actividades son: planificar la calidad, realizar el aseguramiento de calidad, realizar el control de calidad.

\section{Gestión de los recursos}

Implica acciones para garantizar la existencia en temporalidad, cantidad y calidad, de los recursos necesarios para asegurar el cumplimiento de las líneas base del proyecto. Las actividades son: desarrollar el plan de recursos, adquirir el equipo del proyecto, desarrollar el equipo del proyecto, dirigir el equipo del proyecto.

\section{Gestión de las comunicaciones del proyecto}

Gestiona los medios requeridos para asegurar que las comunicaciones se den en condiciones adecuadas. Implica planear quién, cómo y cuándo comunica y a quién comunica, además de definir los canales de comunicación, los medios de almacenamiento y resguardo de la totalidad de los documentos y el conocimiento que genere el proyecto. Las actividades son: identificar a los interesados (Stakeholders), planificar las comunicaciones, distribuir la información, informar el desempeño y gestionar las expectativas de los interesados.

\section{Gestión de los riesgos del proyecto}

Determina cómo planificar la gestión de riesgos, identificar los riesgos, realizar el análisis cualitativo de riesgos, realizar el análisis cuantitativo de riesgos y planificar la respuesta a los riesgos y su monitoreo. Las actividades son: planificar la gestión de riesgos, identificar los riesgos, realizar el análisis cualitativo de riesgos, realizar el análisis cuantitativo de riesgos, planificar la respuesta a los riesgos y monitorizar y controlar los riesgos.

\section{Gestión de las adquisiciones del proyecto}

Define cómo planificar las adquisiciones, efectuar las adquisiciones, administrar las adquisiciones y cerrar las adquisiciones. Las actividades son: planificar las adquisiciones, efectuar las adquisiciones, administrar las adquisiciones y cerrar las adquisiciones.

\section{Gestión de interesados del proyecto}

Permite la identificación, el análisis y el manejo de los interesados. Implica que a lo largo de los procesos de iniciación, planificación, ejecución, seguimiento y control y del cierre mismo, se esté realizando de forma permanente la identificación, el análisis y el manejo de los interesados.

Fuente. Elaboración a partir de $\mathrm{PMBOK}^{\circledR}$. 
- Metodología marco lógico: herramienta fuerte en la formulación del proyecto y en el análisis de precondiciones. Instituto Latinoamericano y del Caribe de Planificación Económica y Social (ILPES). Método que permite realizar el diseño e identificación, la definición, la valoración, la ejecución y supervisión y hasta la evaluación. Dando respuesta a preguntas como ¿cuál es el problema?, ¿qué debemos y cómo debemos hacer?, etc. En cualquier caso se recomienda complementar esta metodología con el $\left(\mathrm{PMI}^{\circledR}\right)$.

\section{PROPOSICIÓN DE PROYECTOS}

A continuación se hace una lista finita y modesta de elementos que pueden ser foco de oportunidad para solucionar problemas en una organización o sociedad y que de seguro son atractivos para el desarrollo integral de las instituciones y de las personas.

\subsection{Proyecto de Responsabilidad Social Empresarial (RSE)}

Es posible que su empresa tenga la madurez necesaria y sea momento de hacer alusión a este tema que no sólo está de moda, sino que además es una necesidad latente de la sociedad, pues determina un alto grado de compromiso y efectos sobre los colaboradores de la organización, los clientes, la honestidad empresarial, la naturaleza y la sociedad menos protegida. Un proyecto de esta envergadura tiene trascendencia de alto impacto en el tiempo.

\subsection{Proyecto de hacer un análisis estructural del sector estratégico}

Una herramienta propuesta por Luis Fernando Restrepo Puerta y Hugo Alberto Rivera, en su libro "Análisis estructural de sectores estratégicos", en el cual proponen varios análisis que permiten identificar a una entidad su condición frente al sector económico al que pertenece. Va desde la evaluación de las condiciones de su producto, con sus diversas formas, hasta la evaluación de la competencia, el análisis del mercado, el análisis financiero, etc. Es una herramienta probada en muchos tópicos y que está generando documentación disponible para el mercado.

\subsection{Proyecto TOC (Theory of Constraints)}

Mediante esta herramienta una empresa pasa de ser una simple generadora de renta, a ser una fábrica de tejido humano. La Teoría de Restricciones Theory of Constraints (TOC), diseñada por Goldratt al principio de los ochenta, es una alternativa que dignifica al hombre. Realiza una trasformación trasversal a la organización, la armoniza y le da sinergias que trascienden sobre las expectativas económicas colectivas.

\subsection{Proyecto de sistemas de gestión}

La gestión de calidad, el modelo estándar de control interno, la gestión ambiental y la gestión de desarrollo administrativo, etc., integran y hacen compatibles los componentes y elementos de las políticas y directrices del Sistema de Desarrollo Administrativo de la empresa, los requisitos de la NTCGP 1000 (Norma Técnica de Calidad para la Gestión Pública) y los lineamientos e instrucciones de la Superintendencia Financiera en relación con el Sistema de Control Interno. Si estos mandatorios no existen, deben crearlos y si existen, deben articularlos.

\subsection{Proyecto de gestión de conocimiento "Gestión de la información"}

La doctora Quintero (2008) ha realizado una interesante proposición para realizar gestión de conocimiento. Los elementos requeridos y propuestos para la gestión son: 
Motivación: (Factor humano). "No sabíamos que era imposible y por eso lo logramos...". Un equipo de trabajo motivado de forma adecuada puede lograr cualquier hazaña;

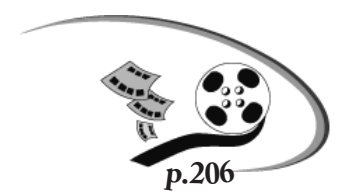

el asunto es lograr conocer a cada uno de sus integrantes y tocar las fibras de cada uno para llegar a movilizarlos. El dinero no es un buen motivador, ya que es un tangible limitado y temporal. Hay que buscar alineación con los elementos que motivan a la persona como persona, como profesional y como ser. Si este primer elemento no se da, la gestión del conocimiento no dará frutos, será un simple aglomerado de datos superfluos. Por tanto no debemos continuar con la gestión del conocimiento si no hay motivación.

Parte de la motivación implica la promoción de valores individuales como honor, lealtad, honestidad, integridad, compasión, amabilidad, altruismo y respeto. Partimos del principio inductivo: si la célula (el ser humano) está bien, el todo (la organización) estará bien.

Generosidad: (Factor humano). Una de las virtudes más escasas en el ser humano es la generosidad y es aun más difícil de hallarla en términos de conocimiento. Lograr que una persona ponga al descubierto sus conocimientos, experiencias, habilidades, virtudes o capacidades artísticas para hacer su trabajo es aún más difícil. Esto es importante porque el objeto de la gestión del conocimiento no es simplemente el de identificar, recoger y organizar el conocimiento existente; en realidad va más lejos. Se trata de facilitar la creación de nuevo conocimiento y apuntalar la innovación a través de la reutilización y apoyo de la habilidad de la gente por medio de organizaciones para lograr un mejor desempeño en la empresa. Esto implica que logremos sacar de nuestro interior ese conocimiento tácito que no sabemos que tenemos y que logremos plasmarlo en documentos que permitan realizar transferencia. Pues bien, esto sólo se logra si existe una adecuada motivación.

Procesos: la formalidad de una acción es la garantía de que se pueda repetir con éxito, por tanto la gestión del conocimiento no puede estar alejada de la existencia de un proceso que la enmarque en la realidad empresarial, por ende debe existir un proceso que la defina, es decir, que contenga la suma de los siguientes elementos: políticas, procesos, procedimientos, formatos, registros, indicadores de gestión e índices de productividad.
Herramientas: las herramientas no garantizan que la gestión del conocimiento se haga de forma adecuada, pero sí garantizan que su difusión sea generalizada. No olvidemos que realizar la entrega de información pobre o aun peor, errada no puede ser controlada por las herramientas usadas para difundirla. Dentro de las alternativas, y sin limitarse a ellas, podemos mencionar el teléfono móvil, el E-mail, el Messenger, la asincronía, los foros y las listas de discusión, el ordenador, Internet, los grupos de investigación, las comunidades de aprendizaje, etc., todas son válidas.

Capital humano: (Factor humano). Nótese que de los cinco elementos mencionados, tres corresponden a temas relacionados con el factor humano. Es que el conocimiento sólo puede ser gestado, contenido, transformado y transferido por los seres humanos. Así, vale la pena ser redundantes y volver a hacer hincapié en el tema de la motivación y la generosidad.

\subsection{Proyecto "Ver la necesidad del desarrollo personal"}

El ser humano, a simple vista, es un ser multidimensional. Eso lo deja ver su desempeño en diferentes roles, como: cónyuge, miembro de una familia, empleado, amigo, miembro de una iglesia o asociación de algo, padre, maestro, hombre de negocios. De hecho es una proposición hecha por Santo Tomás de Aquino, el que el ser humano es un ser multidimensional.

Dentro de las tantas dimensiones del ser se pueden mencionar su dimensión social, su dimensión física, su dimensión psicológica, su dimensión ética, su dimensión intelectual, su dimensión política, su dimensión religiosa, su dimensión económica, entre otras.

La diferencia entre los valores morales y los principios morales, como la abstracción de los primeros sobre los segundos, es que los segundos son una ley inviolable y los primeros la forma de vivirla: Principios: leyes materiales, reglas o normas de conducta que orientan la acción de un ser humano. Son evidentes por sí mismos. Valores: la apropiación de los principios. Cualidad sui generis de un objeto. Los valores son agregados a las características físicas. 
Interiorizando la necesidad de catalizar la dignidad, comprendiendo que se es un ser racional, dotado de libertad, de poder creador y que tiene que además de ser digno, desarrollar su dignidad; comprendiendo que la ética brinda cohesión y coherencia de la multidimensionalidad del ser y esto, a su vez, define el desarrollo apropiado de los roles, podemos establecer que el desarrollo de todas y cada una de las dimensiones del ser, de forma simultánea y notable, garantizan una sociedad funcional; asumiendo que una sociedad funcional sea la suma de condiciones que garantizan que la persona natural o jurídica es ecuánime con sus semejantes y antitéticos, con sus clientes o dependientes, con la sociedad, con el medio y la naturaleza, lo cual redunda en gallardía. "Nuestra recompensa se encuentra en el esfuerzo y no en el resultado. Un esfuerzo total es una victoria completa" (Mahatma Gandhi). Redundando con lo dicho, se establece que un ser se desarrolla, entre otros, en el nivel de:

Dimensión social: tomándola como la maestría con la que un ser logra relacionarse con los demás seres. Basado en su capacidad de comunicación, fundada en la cualidad de escuchar, entender, ratificar el haber entendido y finalmente el ser entendido. Adicional a la práctica de cualidades asertivas y empáticas con los demás. Esto partiendo del principio de una dimensión social sana.
El desarrollarse socialmente implica que sus relaciones con los demás impliquen desarrollar la capacidad de comprenderlos y lograr ser comprendido por los demás; el lograr garantizar en las relaciones un resultado ganar/ganar y el maximizar resultados, siendo eficiente, eficaz y efectivo, gracias a la sinergización.

Dimensión física: definida como la corporeidad del ser, es la condición física de las personas. Hace referencia al poseer un cuerpo, un mecanismo de proyección sobre el medio físico. Siendo cada uno una unidad individual que se engrana con un todo, lo cual redunda en interdependencias.

Físicamente el ser se desarrolla cuando logra un equilibrio entre su cuerpo y su mente, esto es el minimizar los daños a la materia, el cuidarla y preservarla con el objeto de lograr longevidad. Entiéndase por longevidad no sólo la dilatación en años de la existencia, sino además su dignidad.

Dimensión psicológica: está dada por la cualidad diferenciadora del ser humano frente a los demás seres vivos de desarrollar su mente, su pensamiento, haciendo posible percibir cosas abstractas e incorpóreas

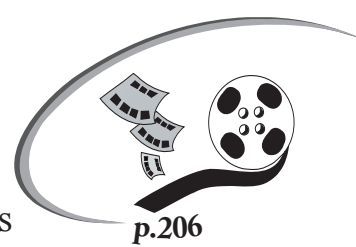
(tabla 2).

Tabla 2. Dimensión psicológica

\begin{tabular}{|c|c|c|c|c|}
\hline Desesperación & Preocupación & Entusiasmo & Sentirse traicionado & Sentirse afortunado \\
\hline Aburrimiento & Confusión & Encanto & Ira & Sentirse capaz \\
\hline Aflicción & Culpabilidad & Energía & Irritación & Sentirse dominado \\
\hline Agotamiento & Curiosidad & Enfado & Lástima & Sentirse herido \\
\hline Agresividad & Debilidad & Enojo & Miedo & Interés \\
\hline Alegría & Decisión & Dolor & Optimismo & Soledad \\
\hline Alivio & Depresión & Envidia & Orgullo & Sorpresa \\
\hline Amor & Desamparo & Esperanza & $\mathrm{Paz}$ & Susto \\
\hline Angustia & Desánimo & Excitación & Pena & Temor \\
\hline Ánimo & Desasosiego & Fastidio & Perplejidad & Tensión \\
\hline Ansiedad & Desazón & Felicidad & Pesimismo & Timidez \\
\hline Aprecio & Desconfianza & Frustración & Confianza & Tranquilidad \\
\hline Arrepentimiento & Desdicha & Hostilidad & Rabia & Tristeza \\
\hline Cansancio & Abatimiento & Impotencia & Rebeldía & Vergüenza \\
\hline Cariño & Determinación & Indecisión & Relajación & Tranquilidad \\
\hline Cautela & Desilusión & Inquietud & Satisfacción & Tristeza \\
\hline Celos & Disgusto & Inspiración & Seguridad & Vergüenza \\
\hline
\end{tabular}

Fuente. Elaboración del autor. 
La psicología desarrollada le permite al hombre tomar control de sus emociones, sentimientos y hasta limitaciones, para lograr disfrutar de aquellos que son positivos y convertir en catalizadores los negativos, es decir, siempre lograr utilidad de las circunstancias.

Dimensión ética: enmarcada en las actitudes o los actos con calificación de buena moral y virtud, denotados por el adecuado cumplimiento del deber, llegando a ser feliz. Todo esto circunscrito a las limitaciones de una determinada sociedad, cultura y época.

Desarrollar la dimensión ética de los seres redunda en la armonía coherente y cohesiva de la sociedad. Es salud social.

Dimensión intelectual: hace referencia a la capacidad que tiene el ser de abstraer, apropiar e interiorizar conocimiento para adaptarse al entorno o para adaptarlo a sus necesidades. Implica un derroche de acciones que permiten recrear, crear, desarrollar, innovar, emular, transformar todo o algo.

El desarrollo intelectual implica paz y armonía, pues sobre la conciencia del por qué de las cosas, el conocer el principio activo de todo, implica la capacidad de predecir efectos y preparase para sobreponerse a los mismos de forma controlada. El conocimiento hace al ser libre de limitaciones mezquinas.

Dimensión política: es la capacidad que tiene el ser de comprender, respetar, subordinarse, violar, transformar, desarrollar o mejorar la delimitación de los condicionados que rigen su capacidad de relacionarse con los demás seres. Es la cualidad que le permite humanizarse, socializarse, crear cohesión. Se desarrolla cuando se reconoce al individuo en la sociedad y a través de la misma logra compartir su identidad y desarrollarla, desplegar sus capacidades de comunicación, definir costumbres, precisar cultura.

El desarrollo de la dimensión política permite que el ser se relacione en condiciones enriquecedoras, dando herramientas de seguimiento y control.

Ahora bien, si el ser es la unidad de la familia y esta es la unidad de la sociedad, podemos afirmar que el desarrollo del ser implica el de la familia, y el de la sociedad. Si el ser humano es un ser multidimensional, con principios morales, dignidad y ética y además se desarrolla de forma integral, lo que vamos a obtener es un ser integrado a la sociedad, responsable de su cuerpo, sus actos y de sus relaciones, que se preocupa por comprender su entorno y gobernar o ser gobernado.

Por lo tanto, el desarrollo del ser debe ser integral. Esto es, que sus relaciones con los demás estarán implicando desarrollar la capacidad de comprenderlos y lograr ser comprendidos por los demás; garantizar en las relaciones un resultado ganar/ganar y maximizar resultados, siendo eficiente, eficaz y efectivo gracias a la sinergización; logrando un equilibrio entre su cuerpo y su mente; teniendo control de sus emociones, sentimientos y hasta limitaciones; teniendo armonía coherente y cohesiva con la sociedad; y logando vivir en paz y armonía.

Si el individuo se ha desarrollado como persona y logra ser feliz, estará garantizado que su desempeño en los diferentes roles ha de ser exitoso. Esto es por ejemplo, un buen cónyuge que no requiere de infidelidad, subyugación o cualquier otro gesto que limite la relación; un buen miembro de una familia que entrega y da sin medida y sin condicionados; un buen empleado que focalizado, organizado y proactivo garantiza eficiencia, eficacia y efectividad en sus acciones y que logra entender, ser entendido, ganar, hacer ganar y sinergizar; un buen amigo que impulsa, levanta y anima a seguir el camino; un buen padre, maestro, hombre de negocios, o cualquier otro rol, que se funda en la gallardía, el honor, la lealtad, la honestidad, la integridad, la compasión, la amabilidad, el altruismo y el respeto para vivir.

Podríamos seguir mencionado proyectos y proyectos, pero con lo ya dicho es posible que si afrontamos temas como un proyecto de liderazgo, un proyecto de implementación de un Balanced Scorecard, un proyecto de seguridad informática, un proyecto de Business Continuity Plan (BCP), un proyecto de sistemas de gestión, etc., tengamos éxito, pues el insumo principal está dado, el capital humano: "talentos, conocimiento explícito y conocimiento tácito". 


\section{REFERENCIAS}

Alcaldía Mayor de Bogotá (2012). Plan de Desarrollo Bogotá Humana 2012-2014. Bogotá, Colombia.

Cámara Oficial de Comercio, Industria y Navegación de Valencia (2010). Responsabilidad Social Empresarial. Recuperado de http://www.rse.org.es/

Alcaldía Mayor de Bogotá (2012). Plan de Desarrollo Bogotá Humana 2012-2014. Bogotá, Colombia.

Cámara Oficial de Comercio, Industria y Navegación de Valencia (2010). Responsabilidad Social Empresarial. Recuperado de http://www.rse.org.es/

Consejo Nacional de Política Económica y Social - CONPES (2004), Documento 3527.,Colombia.

Covey, S. R. (2002). Los 7 hábitos de la gente altamente efectiva: la revolución ética en la vida cotidiana y en la empresa. Barcelona: Paidós.

Departamento Nacional de Planeación (2004). Cadenas Productivas: Estructura, comercio internacional y protección. Recuperado de https://www.dnp.gov.co/Programas/DesarrolloEmpresarial/CadenasProductivas.aspx

Goldratt, E. M. (2001). Cadena crítica: una novela empresarial sobre la Gestión de proyectos. Madrid: Díaz de Santos.

González, Y. I., Joyanes, A. L. (2008). Una propuesta para la profesionalización de la gestión del conocimiento en Colombia. Universidad \& Empresa 7(15), 105-124.

Ministerio de Comercio, Industria y Turismo - MinCIT (2013). Tratados vigentes. Recuperado de http://www.tlc.gov.co/ publicaciones.php?id $=5398$

Project Management Institute (2012). A Guide to the Project Management Body of Knowledge - PMBOK ${ }^{\circledR}$. Guide 5Th Edition. Pennsylvania-USA.

Restrepo, L. F. y Rivera, H. A. (2007). Análisis estructural de sectores estratégicos. Bogotá: Editorial Universidad del Rosario.

Rosenberg y Posner (1979). El marco lógico. Agencia de los Estados Unidos para el Desarrollo Internacional.

Zittlau, J. (2005). Gandhi en la empresa. España: Planeta. 